\title{
A New Algorithm for 3D Profilometry Based on Phase Measurement
}

\author{
Luigi Di Stefano ${ }^{1}$ and Frank Boland ${ }^{2}$ \\ 1 DEIS, University of Bologna \\ Viale Risorgimento 2, 40136 Bologna, Italy \\ 2 EEE, University of Dublin \\ Trinity College, Dublin 2, Ireland
}

\begin{abstract}
This paper describes a new phase extraction algorithm for phase profilometry. The algorithm uses a square wave to demodulate phase and moving averages and comb-shaped filters to extract the phase information from low-frequency. The proposed algorithm is compared with the two major profilometry techniques, namely Fourier domain profilometry and signal domain profilometry based on FIR low-pass filtering.
\end{abstract}

\section{Introduction}

Automated, non-contact 3D optical profilometry gathers ever-increasing attention in industrial applications such as on-line inspection, quality control, gauging of manufactured parts [1]. In such a context active methods based on the projection of structured light are very popular and commercially successful [2]. In this paper we address structured light techniques based on the projection of a $2 \mathrm{D}$ regular grating and phase measurement, i.e phase profilometry techniques, which are particularly attractive among active methods due to the fast acquisition time, very simple optical arrangement and low cost.

In phase profilometry a periodic structured light pattern is projected onto the object and viewed by a camera from an angularly offset position. The typical pattern is a sequence of equally spaced horizontal or vertical dark lines, called "fringes", which are generated projecting a square wave grating. Since the imaged pattern is phase-modulated according to the topography of the object, the extraction of phase information from the image allows reconstruction of the 3D shape. Of the two major phase extraction techniques, one, called Fourier Transform Profilometry (FTP), relies on processing the image in the frequency domain while the other is based on demodulation and convolution operations executed in the real signal domain (we will refer to this technique as Signal Domain Profilometry, SDP). In this paper we present a new signal-domain phase extraction technique which uses a fast demodulation scheme and relies entirely on very simple operators such as moving averages and comb filters. We also compare this new technique with FTP and SDP, addressing adaptiveness to patterns of different frequencies, ability to deal with surfaces of nonuniform reflectivity and computational complexity. 


\section{Phase Profilometry Techniques}

It can be shown that, once a reference plane is selected, the image of the pattern used in phase profilometry can be expressed as

$$
g(x, y)=r(x, y) \sum_{n=-\infty}^{+\infty} A_{n} e^{j\left(2 \pi n f_{0} x+n \phi(x, y)\right)}
$$

while the reference image, i.e. the image obtained projecting the pattern on the reference plane, is given by

$$
g_{0}(x, y)=\sum_{n=-\infty}^{+\infty} A_{n} e^{j\left(2 \pi n f_{0} x+n \phi_{0}(x)\right)}
$$

with $\phi(x, y)-\phi_{0}(x)$, referred to as "phase deviation", being a known function of the object's height distribution with respect to the reference plane. In (1) and (2) $x$ and $y$ are the horizontal and vertical axis, the pattern lines are vertical, $f_{0}$ is the pattern frequency, $A_{n}$ are the Fourier coefficients of the pattern and $r(x, y)$ represents the reflectivity of object's surface. Hence, image rows are expressed as a summation of phase-amplitude modulated harmonics, amplitude modulation due to non-uniform reflectivity and phase modulation embodying the object's $3 \mathrm{D}$ shape. It is worth noting that, since the grating is a square wave, the spectrum of a row contains only the odd harmonics and that the low frequency term is due to $r(x, y)$.

Based on this principle, two major techniques aimed at extracting phase from the imaged pattern have been devised. In FTP [3], [4] the phase profile associated with each image row is evaluated as the angle of the analytic representation of the modulated first harmonic. This is obtained by computing the FFT of the row, filtering out all frequencies outside a narrow band centered at $f_{0}$ and computing the IFFT. In SDP [5] each image row is multiplied by $e^{-j 2 \pi f_{0} x}$ so as to shift the spectrum towards the left by $f_{0}$. Thus, the phase signal associated with the first harmonic moves to low-frequency and is extracted through a low-pass FIR filter. Phase is evaluated as the angle of the complex signal extracted by the filter. With both techniques all the processing steps are applied also to the reference image in order to evaluate phase deviation. SDP yields a substantial reduction of computing time with respect to FTP [5].

\section{A New Phase Extraction Algorithm}

We have developed a new phase extraction algorithm based on moving the phase information to low-frequency and extracting it by means of signal-domain filters. Let us consider the two periodic real functions $c_{1}(x)$ and $c_{2}(x)$ :

$$
c_{1}(x)=\sum_{n=-\infty}^{+\infty} C_{n} e^{j 2 \pi n f_{0} x}
$$




$$
c_{2}(x)=c_{1}\left(x-p_{0} / 4\right)=\sum_{n=-\infty}^{+\infty} C_{n} e^{j 2 \pi n f_{0} x} e^{-j n \pi / 2}=\sum_{n=-\infty}^{+\infty} \tilde{C}_{n} e^{j 2 \pi n f_{0} x}
$$

and multiply the row $y=\bar{y}$ of the deformed pattern image by $c_{1}(x)$ and $c_{2}(x)$ :

$$
\begin{aligned}
& q_{r}(x, \bar{y})=r(x, \bar{y})\left(\sum_{n=-\infty}^{+\infty} A_{n} e^{j\left(2 \pi n f_{0} x+n \phi(x, \bar{y})\right)}\right)\left(\sum_{n=-\infty}^{+\infty} C_{n} e^{j 2 \pi n f_{0} x}\right) \\
& q_{i m}(x, \bar{y})=r(x, \bar{y})\left(\sum_{n=-\infty}^{+\infty} A_{n} e^{j\left(2 \pi n f_{0} x+n \phi(x, \bar{y})\right)}\right)\left(\sum_{n=-\infty}^{+\infty} \tilde{C}_{n} e^{j 2 \pi n f_{0} x}\right)
\end{aligned}
$$

If the mean value of $c_{1}(x)$ is zero, the low-frequency components of $q_{r}$ and $q_{i m}$, indicated as $\bar{q}_{r}$ and $\bar{q}_{i m}$, can be expressed as

$$
\begin{gathered}
\bar{q}_{r}(x, \bar{y})=2 r(x, \bar{y}) \sum_{n=-\infty}^{+\infty}\left|A_{n}\right|\left|C_{n}\right| \cos \left(\alpha_{n}+n \phi(x, \bar{y})-\gamma_{n}\right) \\
\bar{q}_{i m}(x, \bar{y})=2 r(x, \bar{y}) \sum_{n=-\infty}^{+\infty}\left|A_{n}\right|\left|C_{n}\right| \cos \left(\alpha_{n}+n(\phi(x, \bar{y})-\pi / 2)-\gamma_{n}\right)
\end{gathered}
$$

where $\alpha_{n}$ and $\gamma_{n}$ are the angles of the complex coefficients $A_{n}$ and $C_{n}$. If a simple smoothing operator such as a moving average is applied to the image prior (5) and (6) in order to attenuate the higher harmonics in $g(x, \bar{y})$, the dominant term in $\bar{q}_{r}$ and $\bar{q}_{i m}$ is that associated with the main harmonic:

$$
\begin{gathered}
\bar{q}_{r}(x, \bar{y}) \cong 2 r(x, \bar{y})\left|A_{1} \| C_{1}\right| \cos \left(\alpha_{1}+\phi(x, \bar{y})-\gamma_{1}\right) \\
\bar{q}_{i m}(x, \bar{y}) \cong-2 r(x, \bar{y})\left|A_{n} \| C_{n}\right| \sin \left(\alpha_{1}+\phi(x, \bar{y})-\gamma_{1}\right)
\end{gathered}
$$

Hence, phase information is embedded into the angle of the low-frequency complex signal

$$
\bar{q}(x, \bar{y})=\bar{q}_{r}(x, \bar{y})-j \bar{q}_{i m}(x, \bar{y}) \cong 2 r(x, \bar{y})\left|A_{1}\right|\left|C_{1}\right| e^{\alpha_{1}+\phi(x, \bar{y})-\gamma_{1}} .
$$

Since execution of the same procedure on the reference image yields

$$
\bar{q}_{0}(x, \bar{y}) \cong 2 r(x, \bar{y})\left|A_{1} \| C_{1}\right| e^{\alpha_{1}+\phi_{0}(x)-\gamma_{1}}
$$

the phase deviation can be extracted by subtracting the angle of $\bar{q}_{0}(x, \bar{y})$ from the angle of $\bar{q}(x, \bar{y})$.

Once shown that the phase information can be moved to low-frequency by multiplying the image by two generic periodic functions, a suitable choice for these functions is a square wave swinging between -1 and +1 . Thus, demodulation requires no more than proper manipulation of pixel signs. The step following demodulation is extraction of the low-frequency spectrum, the main task being rejection of the unwanted spectra which are generated in the demodulation process. Since these are centered at specific harmonics of the grating frequency, we 
propose to apply first a comb-shaped filter in order to reject selectively unwanted harmonics and then a moving average to obtain the required low-pass behavior. The proposed technique is potentially faster than SDP since the demodulation cost is practically negligible and only very fast filters such as comb or modified comb filters [6] are used.

\section{Simulation Analysis}

We use simulations to discuss adaptiveness of phase extraction techniques with respect to changes of the fringe frequency. Adaptiveness is a key requirement since coarse fringe patterns are effective for measuring coarse surface variations while fine patterns are suited to the measurement of fine surface details [7]. In our analysis we will take into account speed of design, accuracy and computational complexity. Simulation methodology and parameters are as follows. Line size is set to 256 pixel. Assuming uniform reflectivity, the image of a one-dimensional fringe pattern is phase-modulated based on a trapezoidal phase profile with 0.15 pixel slope. We simulate projection of a fine fringe pattern $\left(f_{0}=0.125 \mathrm{pixel}^{-1}\right.$, 32 fringes per line) and of a coarse one $\left(f_{0}=0.625\right.$ pixel $^{-1}, 16$ fringes per line).

First we consider the fine pattern and the new technique. According to previous section, the line is smoothed using a 3-point moving average. Since reflectivity is uniform, we subtract the mean value before demodulation in order to get rid easily of the DC component. Then, demodulation is carried out by proper manipulation of pixel signs. Since after demodulation the main unwanted spectra are centered at frequencies $\mp 2 f_{0}$, we apply the comb filter capable of eliminating these frequencies, given by $y_{k}=x_{k}+x_{k-2}$. As a result, the low-frequency signal can be easily extracted by a 9 -points moving average. The left plot of Fig. 1 provides a proof of the feasibility of the method: the extracted phase profile (solid line) closely approximates the target phase profile (dashed line); the mean absolute phase error is $31 \mathrm{mrad}$. As far as FTP is concerned, we implemented the frequency domain filter by hanning window in order to reduce ripple. With regard to SDP, we use the 9 taps FIR filter proposed in [5]. Phase profiles extracted by FTP and SDP, not shown here, can be considered equivalent to that in the left plot of Fig. 1, phase errors being 29 mrad for FTP and 35 mrad for SDP.

We consider now the coarse fringe pattern. Adapting our technique to this new pattern consists in tuning the comb-shaped filter so as to eliminate frequencies $\mp 2 f_{0}, \mp 4 f_{0}, \mp 6 f_{0}$ from the demodulated signals. Since the filter $y_{k}=$ $x_{k}+x_{k-2}$ eliminates frequencies $\mp 4 f_{0}$ while the filter $y_{k}=x_{k}+x_{k-4}$ eliminates frequencies $\mp 2 f_{0}, \mp 6 f_{0}$, the cascade of the two gives the modified comb filter suited to the coarse fringe pattern: $y_{k}=x_{k}+x_{k-2}+x_{k-4}+x_{k-6}$. Thus, the design process is quite simple and fast. Moreover, computational complexity remains practically as low as in the fine pattern case. In fact the new filter requires just one more subtraction per point since it can be split into two independent filters, running on even and odd pixels, computed through the recursive relation $y_{k}=y_{k-2}+x_{k}-x_{k-8}$. The phase profile extracted with the coarse pattern is 

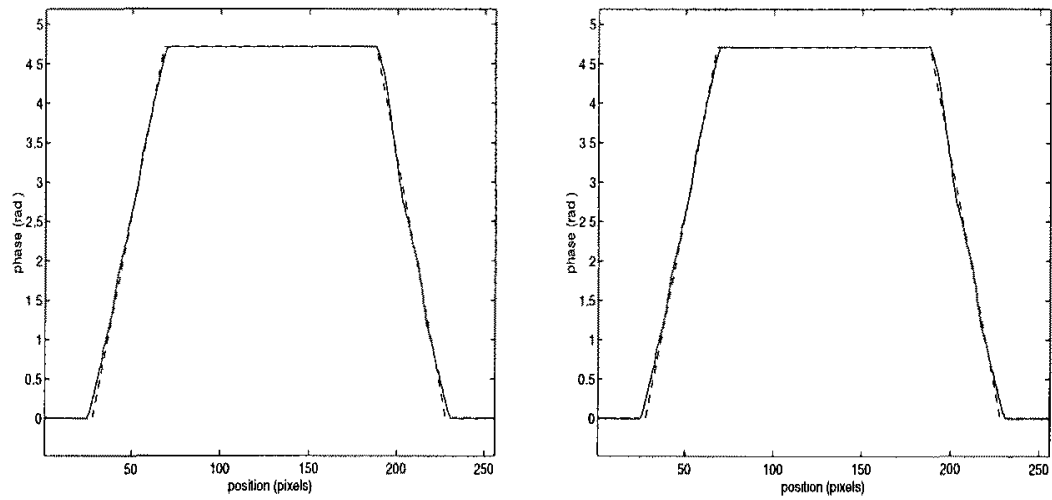

Fig. 1. Phase profiles extracted by the new method
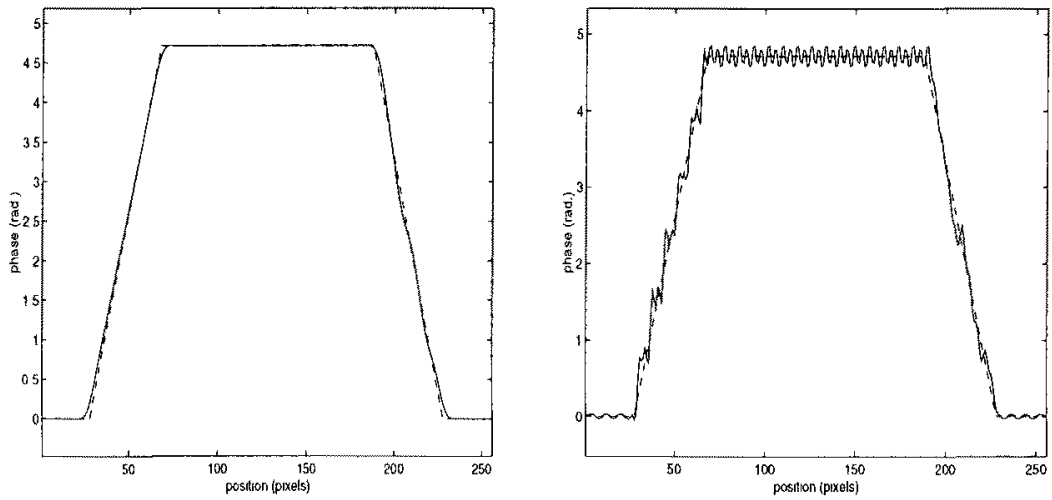

Fig. 2. Coarse pattern: phase profiles extracted by FTP (left) and SDP (right)

shown in the right plot of Fig. 1, the mean absolute phase error being $40 \mathrm{mrad}$. Adapting FTP to the new pattern requires just halving the size of the window and centering it at the new frequency. Since computation time is largely dominated by the FFT-IFFT pair, complexity is nearly independent of the pattern frequency. On the other hand, a suitable FIR filter must be designed from scratch to adapt SDP to a new pattern frequency. Since in this case the main unwanted spectra are close to DC, a quite sharp transition from passband to stopband is needed to extract the low-frequency spectrum. This makes the design extremely problematic. We have discussed this issue in detail in [8] addressing 9 taps minimax FIR filters; here we use the best 9 taps filter shown in [8]. Profiles extracted by FTP and SDP are plotted in Fig. 2, phase error being $45 \mathrm{mrad}$ with FTP and $96 \mathrm{mrad}$ with SDP.

Thus, with both FTP and our technique one can easily adapt the procedure to a different fringe pattern, no practical variation in the computational complexity is associated with the frequency change, and the accuracy with a 
coarse pattern remains rather good compared to that of the fine pattern case. Conversely, adapting SDP implies the carrying out of a FIR filter design process which turns out to be very critical in case of a coarse pattern. Moreover, if in this case the order of the filter is low, SDP is significantly less accurate. The only way to improve SDP accuracy with a coarse pattern is to increase the order of the filter. We had to increase the number of taps up to 21 to push the error within $50 \mathrm{mrad}$ (i.e. $45 \mathrm{mrad}$ ). Therefore, even though with a coarse pattern SDP may provide an accuracy level similar to that of the other two techniques, this implies a substantial increase of the computational load.

We have assumed uniform reflectivity, but this is not the case of most real images. This would introduce errors with every phase extraction technique since the low-frequency reflectivity spectrum may overlap with the spectrum to be extracted. In addition, with SDP the filter design becomes even more complicated since a much sharper transition is required to attenuate the reflectivity spectrum, which is shifted to $-f_{0}$ by demodulation. In the following section we address this issue in the context of $3 \mathrm{D} \mathrm{PCB}$ inspection.

\section{Experimental Results}

We have investigated the use of phase-profilometry for 3D PCB inspection [8], [9], that consist in reconstructing the $3 \mathrm{D}$ shape of the solder paste printed on SMT (Surface Mount Technology) component pads before component placement. The left image in Fig. 3 shows a circular pad on the PCB with the projected fringe pattern. Image size is $256^{*} 256$ pixels and fringe frequency is $0.0625 \mathrm{pixel}^{-1}$.
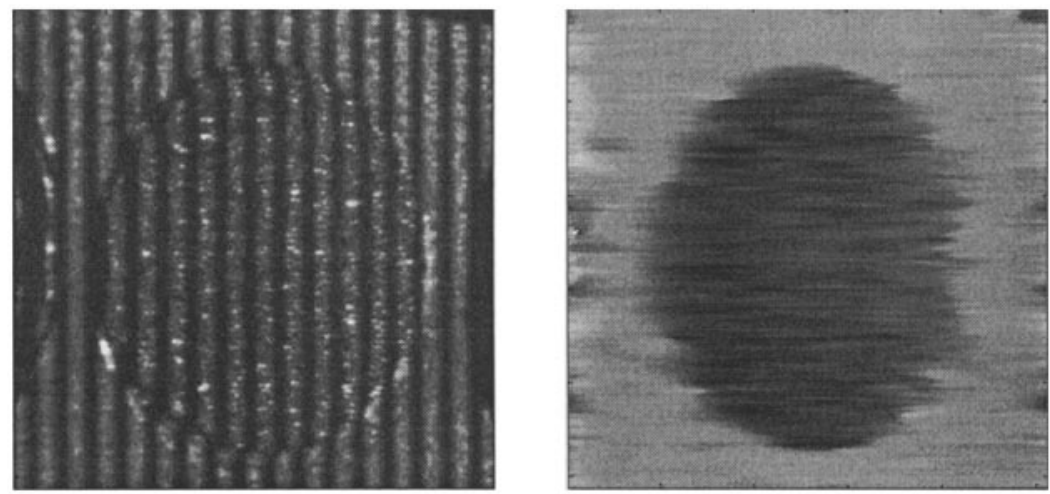

Fig. 3. Original image and phase map extracted by FTP

With FTP we deal with the potential overlap between spectra by reducing the size of the filter. Although this requires some tuning, it is still an easy and rapid process. The phase map extracted from the pad image using FTP is shown in the right image of Fig. 3, darker points representing higher phase deviations 

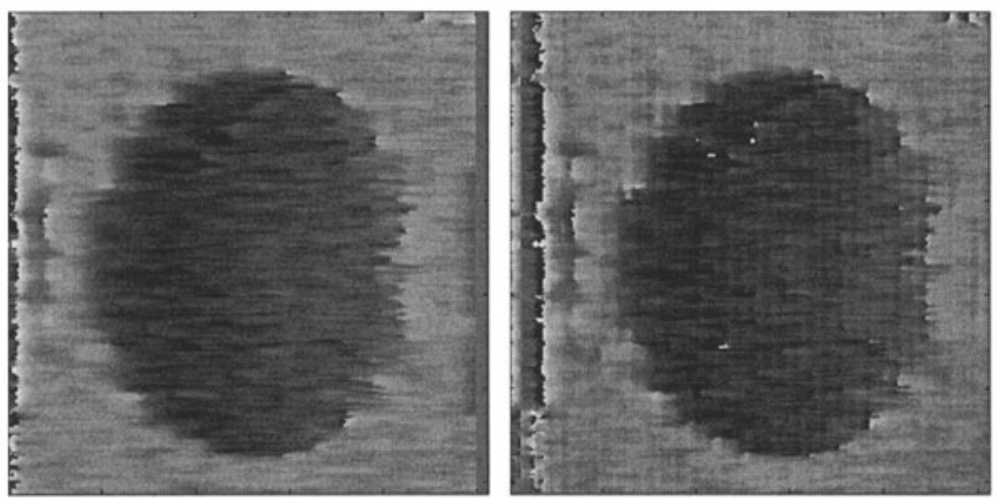

Fig. 4. Phase map extracted by our technique and by SDP

(i.e. heights). As far as the proposed technique is concerned, the comb filter should now attenuate all the fringe frequency harmonics. Thus, we cascade the filter $y_{k}=x_{k}+x_{k-8}$ to the filter for the coarse pattern used in the simulations; this yields the modified comb filter: $y_{k}=x_{k}+x_{k-2}+x_{k-4}+x_{k-6}+x_{k-8}+$ $x_{k-10}+x_{k-12}+x_{k-14}$. Since the filter can be computed through the relation $y_{k}=y_{k-2}+x_{k}-x_{k-16}$, the computational load is the same as that of the filter used in the simulations. The phase map extracted using our technique is shown in the left image of Fig. 4.

Due to unavailability of a suitable high-accuracy metrology equipment, we cannot assess quantitatively these results. However, qualitative judgment provides useful hints with respect to comparative analysis of the addressed technique. In this context, the clear contrast between the flat background area and the circular shaped object region shows that both techniques allow neat detection of the elevation with respect to $\mathrm{PCB}$ surface associated with solder paste on pads. Use of SDP for PCB inspection requires the FIR filter to be redesigned, with a much sharper transition required to deal with nonuniform reflectivity. As a result, the filter order must be increased to 29 to obtain results qualitatively similar to those of the other two techniques. The phase map extracted by the 29 taps filter is shown in the right image of Fig. 4.

Eventually, we have measured computation times $(90 \mathrm{MHz}$ Pentium PC, image size: $256^{*} 256$ ) in order to compare complexity of the addressed techniques. We have considered the time required to extract the complex signal embodying phase deviation $\left(T_{1}\right)$, which is peculiar of a given technique, and the total time for obtaining the phase map $(T)$, obtained by adding to $T_{1}$ the constant time ( $\left.T_{2}=0.4441 \mathrm{secs}\right)$ required to evaluate the angle and subtract the reference. As already observed, with both FTP and the new technique the computational load is nearly constant. Hence, using the procedures developed for PCB inspection, we have measured $T_{1}=1.1156 \mathrm{secs}$ for FTP and $T_{1}=0.0537 \mathrm{secs}$ for our technique. Conversely, with SDP we have evaluated $T_{1}$ using the 9,21 and 29 taps filters (suited respectively to the fine pattern, coarse pattern, coarse pattern 
and nonuniform reflectivity), measuring respectively $0.1992 \mathrm{secs}, 0.4173 \mathrm{secs}$ and 0.5390 secs. Hence, our algorithm is, roughly, 20 times faster than FTP and, taking the average of the times for the 3 filters, 7 times faster than SDP. However, due to the substantial value of $T_{2}$ the actual speedup is lower. Nonetheless, evaluation of $T$ shows that our algorithm renders profilometry 3 times faster than using FTP, while the speedup with respect to SDP can range from $30 \%$, as in the case of fine patterns, to almost $100 \%$, as in the case of coarse patterns and nonuniform reflectivity.

\section{Conclusion}

We have presented a new phase extraction algorithm for phase profilometry. The algorithm relies on a fast demodulation scheme and very simple DSP operators such as comb filter and moving averages. We have also compared it with FTP and SDP. The new technique's advantage with respect to FTP is speed since it renders profilometry roughly 3 times faster. With respect to SDP, the main advantage is adaptiveness. In fact, while one can easily tune a comb-shaped filter to reject different frequencies, SDP requires complete re-design of the low-pass FIR filter. This task becomes very critical when the fringes frequency is low and/or reflectivity is nonuniform, with satisfactory results attainable only at the expense of a significant increase of the filter complexity. Therefore, the proposed technique is also notably faster than SDP in the case of coarse patterns and/or nonuniform reflectivity.

\section{References}

1. D. Poussart and D. Laurendau. 3-D sensing for industrial computer vision. in J. Sanz, editor, Advances in Machine Vision, Springer-Verlag, 1989.

2. P. Besl. Active, optical range imaging sensors. Machine Vision and Applications, Vol.1, 1988.

3. M. Takeda, H. Ina and S. Kobayashi. Fourier-transform method of fringe-pattern analysis for computer-based topography and interferometry. Journal of the Optical Society of America, Vol. 72, No. 1, 1982.

4. M. Takeda and K. Mutoh. Fourier transform profilometry for the automatic measurement of 3-D object shapes. Applied Optics, Vol. 22, No. 24, 1983.

5. S. Tang and Y. Hung. Fast profilometer for the automatic measurement of 3-D object shapes. Applied Optics, Vol. 29, No. 20, 1990.

6. E. Cunningham. Digital Filtering: an introduction. Houghton Mifflin, 1992.

7. G. Sansoni, L. Biancardi, U. Minoni and F. Docchio. A Novel Adaptive System for 3-D Optical Profilometry Using a Liquid Crystal Light Projector. IEEE Transactions on Instrumentation and Measurement, Vol. 43, No. 4, 1994.

8. L. Di Stefano and F. Boland. Three-Dimensional Inspection of Printed Circuit Boards Using Phase Profilometry. In Proceedings of EUSIPCO-96, Trieste, 10-13 September, 1996.

9. L. Di Stefano and F. Boland. Solder paste inspection by structured light methods based on phase measurement. In SPIE Proceedings, Vol. 2899, 1996. 